\title{
Status and Location of Hot Spots of Stem and Pod Rot of Groundnut in Karnataka, India
}

\author{
Poornima $^{1^{*}}$, Gururaj Sunkad ${ }^{2}$ and H. Sudini ${ }^{2}$ \\ ${ }^{1}$ Department of plant pathology, AICRP (Sunflower) MARS, Raichur \\ ${ }^{2}$ Department of plant pathology, University of Agricultural Sciences, Raichur- 584104 , \\ Karnataka, India \\ ${ }^{2}$ International Crops Research Institute for the Semi-Arid Tropics Patencheru - 502324 \\ Telangana, India \\ *Corresponding author
}

\section{A B S T R A C T}

\begin{tabular}{|l|}
\hline Ke y w or d s \\
Groundnut, Stem \\
and pod rot, \\
S.rolfsii, Survey, \\
Hot spot \\
\hline Article Info \\
\hline $\begin{array}{l}\text { Accepted: } \\
\text { 26 June } 2018 \\
\text { Available Online: } \\
\text { 10 July 2018 }\end{array}$ \\
\hline \hline
\end{tabular}

\begin{abstract}
A survey was conducted in major groundnut growing areas of Karnataka during kharif 2013 and kharif 2014 to identify hot spots of stem and pod rot of groundnut. A total of 80 different locations of eight districts of Karnataka were covered during the survey. During kharif 2013, the highest stem rot $(62.80 \%)$ and pod rot $(73.67 \%)$ was recorded in Chitradurga district followed by Tumkur district which recorded stem rot incidence of $42.59 \%$ and pod rot of $40.70 \%$. Least incidence of stem rot (17.19\%) was recorded in Koppal district and pod rot (8.33\%) in Gadag district. During kharif 2014, highest incidence of stem rot $(57.83 \%)$ and pod rot $(71.63 \%)$ was observed in Chitradurga district followed by Tumkur district recorded stem rot $(46.09 \%)$ and pod rot $(48.34 \%)$. Least stem rot of $(17.23 \%)$ and pod rot of $(8.58 \%)$ was recorded in Koppal district. Among surveyed districts, Chitradurga showed highest mean incidence of stem rot $(60.31 \%)$ and pod rot $(72.65 \%)$ followed by Tumkur (44.34\% and $44.52 \%$, respectively). Hence, Chitradurga and Tumkur districs were considered as hot spot for stem and pod rot of groundnut in Karnataka. However, the lowest mean incidence was observed in Koppal (stem rot - $17.21 \%$ and pod rot $-11.29 \%$ ) followed by Gadag (stem rot $-17.93 \%$ and pod rot $-10.79 \%$ ) and Kalaburgi (stem rot $-21.31 \%$ and pod rot $-18.45 \%$ ).
\end{abstract}

\section{Introduction}

Groundnut (Arachis hypogaea L.), is an important leguminous oilseed crop, belonging to the family Fabaceae (Nordern et al., 1982). Groundnut is called as the 'king' of oilseeds. It is one of the most important food and cash crops of our country. Groundnut is grown under rainfed and as well as irrigated condition. Groundnut is grown in 254.60 lakh ha with a production of 453.08 lakh tons and productivity of $1780 \mathrm{~kg} / \mathrm{ha}$ in the world
(Anon., 2014). It is grown nearly in 100 countries worldwide and the major groundnut producing countries are China, India, Nigeria, USA, Indonesia and Sudan. In India, groundnut is the principal oilseed crop and is grown in an area of 52.50 lakh ha with an annual production of 94.72 lakh tons and a productivity of $1804 \mathrm{~kg} \mathrm{ha}^{-1}$ (Anon., 2014). Major groundnut growing states of India include Gujarat, Maharastra, Karnataka, Tamil Nadu, Telangana, Andhra Pradesh. Among the states, Andhra Pradesh, Telangana and Gujarat 
contribute to more than half the crop area in our country (Anon., 2014). Six States namely Andhra Pradesh, Gujarat, Karnataka, Maharashtra, Rajasthan and Tamil Nadu account for about $90 \%$ of the total groundnut area of the country. Andhra Pradesh and Gujarat contribute $>55 \%$ of the total area and production of groundnut (Anon., 2014). However, the yield of groundnut is reducing day by day because of various biotic and abiotic factors. Among the biotic factors diseases are the most important constraints for the yield reduction. The majority is caused by fungi and several of them are yield reducers in certain regions and seasons. Among the soil borne diseases stem and pod rot caused by Sclerotium rolfsii Sacc. is emerging as a major problem and has become an economically important soil borne pathogen. Symptoms of stem and pod rot disease are initially there will be yellowing and partial or complete wilting of the stem or one or more branches. In advanced stage of the disease, white mycelial growth at the junction of stem and soil, spreads over the soil and the basal canopy of the plant. The sclerotia of the size and colour of mustard seeds, appear on the infected area as the disease develops. The entire plant may be killed or only two or three branches may be affected. Infected pods were completely covered with white mycelial growth and in severe cases rotting of pod were observed (Plate 1). During the recent years this disease causes significant losses in groundnut growing areas of Karnataka. Hence, the present study was conducted with an objective to locate hot spots for stem and pod rot of groundnut in Karnataka during kharif 2013 and 2014.

\section{Materials and Methods}

Roving survey was taken up from major groundnut growing places of Karnataka during kharif, 2013 and kharif, 2014 between 90 to 110 days old crop to know the incidence of stem and pod rot of groundnut. Samples were collected from eight districts and from each district predominant groundnut growing areas were chosen for sample collection and assessment of disease. All samples showing typical symptoms of stem rot and pod rot disease were collected in butter paper bags brought to the laboratory and stored for further isolation studies. For recording the disease, five spots in each field and 100 plants at each spot were selected randomly. The total number of plants presents and number of plants showing stem rot symptoms due to Sclerotium rolfsii at each spot were counted and recorded. Later, the per cent stem rot incidence in these locations was calculated using following formula.

Stem rot incidence $(\%)=$

Number of stem rots infected plants x 100

Total number of plants

Recording pod rot incidence, dry pods of five plants were plucked randomly from each spot and total number of pods per plant and number of pods infected by $S$. rolfsii pathogen were counted and recorded. Later, the per cent pod rot incidence in these locations was calculated using following formula.

Pod rot incidence $(\%)=$

Number of pods infected/ plant

Total number of pods/ plant

Meantime, the co-ordinates of surveyed fields were also recorded with the help of GPS and details of co-ordinates were listed in Table 1 and Table 2 .

\section{Results and Discussion}

In the present study, an intensive roving survey for stem and pod rot of groundnut was carried out during kharif 2013 and 2014 in major groundnut growing areas of Karnataka 
to get precise information on the distribution and intensity of the disease. The data on survey revealed that, the stem and pod rot incidence varied from locality to locality, because of varieties grown viz., KRG-1, TMV-2 and JL-24, environmental conditions viz., relative humidity, temperature and soil moisture content and during the survey we could able to observe cropping pattern like maize-groundnut, sorghum-groundnut and groundnut-groundnut. Even, it could also be attributed to the existence of variability or pathogenic diversity present in the fungus. This is in agreement with (Kulkarni, 2007) who reported variable incidence of $S$. rolfsii at different localities of Belganvi and Dharwad districts.

The overall mean incidence of stem rot $(62.80 \%)$ and pod rot $(73.67 \%)$ was more during kharif, 2013 compared to kharif 2014 (stem rot-57.83\% and pod rot-71.63\%). This may be due to variations in climatic conditions prevailed during the years at different locations (Fig. 1, 3 and 6).

During kharif 2013, the highest stem rot $(62.80 \%)$ and pod rot $(73.67 \%)$ was recorded in Chitradurga district followed by Tumkur district which recorded stem rot incidence of $42.59 \%$ and pod rot of $40.70 \%$. Least incidence of stem rot $(17.19 \%)$ was recorded in Koppal district and pod $\operatorname{rot}(8.33 \%)$ in Gadag district (Table 3). During kharif 2014, highest incidence of stem rot $(57.83 \%)$ and pod rot $(71.63 \%)$ was observed in Chitradurga district followed by Tumkur district recorded stem rot $(46.09 \%)$ and pod rot $(48.34 \%)$. Least stem rot of $(17.23 \%)$ and pod rot of $(8.58 \%)$ was recorded in Koppal district (Table 4).

The highest mean incidence of stem rot $(64.44 \%)$ and pod rot $(79.99 \%)$ was recorded in Hiriyur taluk followed Challakere $(56.23 \%$ and $65.31 \%$ ) of Chitradurga district (Fig. 7) and Pavagada taluk of Tumkur district
(48.26\% and 47.16\%) (Fig. 8). The least incidence of stem rot $(13.42 \%)$ and pod rot $(1.66 \%)$ were recorded in Kushtagi taluk of Koppal district (Fig. 2). Among surveyed the districts, Chitradurga showed highest mean incidence of stem rot $(60.31 \%)$ and pod rot (72.65\%) followed by Tumkur $(44.34 \%$ and $44.52 \%$, respectively) and Ballari for stem rot $(31.81 \%)$ and Raichur for pod rot $(32.28 \%)$ over two seasons. However, the lowest mean incidence was observed in Koppal $(17.21 \%$ and $11.29 \%)$ followed by Gadag $(17.93 \%$ and $10.79 \%$ ) (Fig. 4) and Kalaburgi (21.31\% and $18.45 \%$ ) (Fig. 5). Data also indicated that the overall mean incidence (Table 5) in the Karnataka state for stem rot was $31.02 \%$ whereas $30.10 \%$ for pod rot.

These results are in conformity with the results of Siddaramaiaha et al., (1979) who reported that Dharwad district recorded an average incidence of $7.8 \%$ stem rot. Ramakrishna and Kolte (1988) found that, average incidence of pod rot of groundnut in India was about 15-30 per cent. Similarly, Pande and Rao, 2000 conducted survey in fields during 1999 rainy season in five districts (Mahaboobnagar, Chittoor, Kurnool, Kadapa and Anantapur) of Andhra Pradesh, two districts (Raichur, Kolar) of Karnataka and one district (Dharmapuri) of Tamilnadu. Highest incidence (29\%) was observed in Raichur district of Karnataka and lowest was observed in Chitthoor district of Andhra Pradesh.

The higher incidence of stem and pod rot disease in Chitradurga and Tumkur districts is due to growing of susceptible variety such as TMV-2 and continuous cropping of groundnut in large area and presence of pathogen over long period. Karunakaran et al., (2013) reported that groundnut is one of the major crop in Chitradurga district where groundnut is grown in large area as a sole crop. 
Table.1 Coordinates of villages selected for survey of stem and pod rot of groundnut during kharif, 2013

\begin{tabular}{|c|c|c|c|c|}
\hline District & Taluk & Village & Longitude & Latitude \\
\hline \multirow[t]{6}{*}{ Raichur } & \multirow[t]{2}{*}{ Raichur } & Chandrabanda & 16.253686 & 77.455222 \\
\hline & & Yapaldinni & 16.279181 & 77.502022 \\
\hline & \multirow[t]{2}{*}{ Lingasugur } & Karadkal & 16.156622 & 76.552197 \\
\hline & & Eachanahal & 16.172592 & 76.437133 \\
\hline & \multirow[t]{2}{*}{ Deodurga } & Galag & 16.270347 & 76.841867 \\
\hline & & Kajjibandi & 16.24630286 & 76.85944303 \\
\hline \multirow{6}{*}{ Koppal } & \multirow{2}{*}{ Koppal } & Muddaballi & 15.281225 & 76.151714 \\
\hline & & Halagera & 15.362364925 & 76.093352717 \\
\hline & \multirow{2}{*}{ Kushtgi } & Kanakoppa & 15.756508 & 76.071914 \\
\hline & & Byalihal & 15.753356 & 76.131053 \\
\hline & \multirow[t]{2}{*}{ Yelburga } & Bandi & 15.732483850 & 76.053142873 \\
\hline & & Rajoor & 15.536357229 & 75.989076582 \\
\hline \multirow[t]{6}{*}{ Ballari } & \multirow[t]{2}{*}{ Ballari } & Kakkabevinahalli & 15.143761 & 77.003622 \\
\hline & & Bommanahal & 15.004289 & 76.980067 \\
\hline & \multirow[t]{2}{*}{ Sirguppa } & Dasapur & 15.427253 & 76.869094 \\
\hline & & Konchigeri & 15.409547134 & 76.872852113 \\
\hline & \multirow[t]{2}{*}{ Kudligi } & Kudligi & 14.925039697 & 76.368576281 \\
\hline & & Sivapura & 14.963372083 & 76.364659925 \\
\hline \multirow[t]{4}{*}{ Gadag } & \multirow[t]{2}{*}{ Gadag } & Mulgund & 15.265431 & 75.523794 \\
\hline & & Chinchalli & 15.287206 & 75.481506 \\
\hline & \multirow[t]{2}{*}{ Ron } & Gajendragad & 15.731642 & 75.958167 \\
\hline & & Goudageri & 15.730825 & 75.988322 \\
\hline \multirow[t]{4}{*}{ Kalaburgi } & \multirow[t]{2}{*}{ Kalaburgi } & Taj Sulthanpur & 17.398089 & 76.816578 \\
\hline & & Kamalapur & 17.571672 & 76.981425 \\
\hline & \multirow[t]{2}{*}{ Aland } & Aland rural & 17.593719 & 76.574661 \\
\hline & & Telkuni & 17.613867 & 76.568906 \\
\hline \multirow[t]{6}{*}{ Yadgir } & \multirow[t]{2}{*}{ Yadgir } & Ramsamudra & 16.775416766 & 77.229311726 \\
\hline & & Hattikuni & 16.844886 & 77.145364 \\
\hline & \multirow[t]{2}{*}{ Shahpur } & Bheemarayangudi & 16.732428 & 76.799144 \\
\hline & & Gundapur & 16.707428925 & 76.628222925 \\
\hline & \multirow[t]{2}{*}{ Shorapur } & Naganoor & 16.686880727 & 76.603037682 \\
\hline & & Khanapur & 16.692208 & 76.601472 \\
\hline \multirow[t]{4}{*}{ Chitradurga } & \multirow[t]{2}{*}{ Challakere } & Challakere rural & 14.289023966 & 76.595261528 \\
\hline & & Nandanhalli & 14.28245 & 76.590558 \\
\hline & \multirow[t]{2}{*}{ Hiriyur } & Yarballi & 14.103679021 & 76.651842727 \\
\hline & & Hartikote & 14.070933 & 76.639233 \\
\hline \multirow[t]{4}{*}{ Tumkur } & Pavgada & Kenchammanahalii & 14.193957693 & 77.004791771 \\
\hline & & Lingadahalli & 14.185172 & 77.041086 \\
\hline & Sira & Dwaranakunte & 14.005609328 & 76.867745423 \\
\hline & & Karehalli & 13.990644 & 76.880889 \\
\hline
\end{tabular}


Table. 2 Coordinates of villages selected for survey of stem and pod rot of groundnut during kharif, 2014

\begin{tabular}{|c|c|c|c|c|}
\hline District & Taluk & Village & Longitude & Latitude \\
\hline \multirow[t]{6}{*}{ Raichur } & \multirow[t]{2}{*}{ Raichur } & Idapnur & 16.022166127 & 77.474291382 \\
\hline & & Jambaldinni & 160458.083 & 772757.344 \\
\hline & \multirow[t]{2}{*}{ Lingasugur } & Neeralkera & 16.143617 & 76.4491 \\
\hline & & Bannigol & 16.65313167 & 77.02761333 \\
\hline & \multirow[t]{2}{*}{ Deodurga } & Karadigudda & 16.42173 & 76.952055 \\
\hline & & Gabbur & 16.296821595 & 77.158786155 \\
\hline \multirow[t]{6}{*}{ Koppal } & \multirow[t]{2}{*}{ Koppal } & Indargi & 15.42052 & 76.3335 \\
\hline & & Ginigera & 15.35728 & 76.26949 \\
\hline & \multirow[t]{2}{*}{ Kushtagi } & Gumgeri & 16.79128667 & 77.13874833 \\
\hline & & Ganganhal & 16.800456 & 77.147858 \\
\hline & \multirow[t]{2}{*}{ Yelburga } & Talakal & 15.382486 & 76.015933 \\
\hline & & Boodagumpa & 15.73214833 & 75.993895 \\
\hline \multirow[t]{6}{*}{ Ballari } & \multirow[t]{2}{*}{ Ballari } & Bevinahalli & 15.145619 & 76.989172 \\
\hline & & Upanayakanahalli & 15.11928127 & 76.25304522 \\
\hline & \multirow[t]{2}{*}{ Sirguppa } & Shanavasapur & 15.407958 & 76.899464 \\
\hline & & Siddaramapura & 15.429553 & 76.854856 \\
\hline & \multirow[t]{2}{*}{ Kudligi } & Bande basapur & 14.950995026 & 76.362529235 \\
\hline & & Hireheggadahal & 14.924419 & 76.358919 \\
\hline \multirow[t]{4}{*}{ Gadag } & \multirow[t]{2}{*}{ Gadag } & Seethalahari & 15.255739 & 75.521219 \\
\hline & & Yalavatti & 15.257733 & 75.513478 \\
\hline & \multirow[t]{2}{*}{ Ron } & Purthageri & 15.735453 & 75.955697 \\
\hline & & Rajur & 15.739311 & 75.938533 \\
\hline \multirow[t]{4}{*}{ Kalaburgi } & \multirow[t]{2}{*}{ Kalaburgi } & Wadagera & 17.577753 & 77.015142 \\
\hline & & Tawargera & 17.415592 & 76.885208 \\
\hline & \multirow[t]{2}{*}{ Aland } & Honhalli & 17.584089 & 76.607333 \\
\hline & & Mamdapur & 17.570892 & 76.609597 \\
\hline \multirow[t]{6}{*}{ Yadgir } & \multirow[t]{2}{*}{ Yadgir } & Anapoor & 16.79128667 & 77.13874833 \\
\hline & & Bandalli & 16.800456 & 77.147858 \\
\hline & \multirow[t]{2}{*}{ Shahpur } & Bhimanahalli & 16.93961736 & 77.19700128 \\
\hline & & Raajaapoor & 16.754075 & 76.79272333 \\
\hline & \multirow[t]{2}{*}{ Shorapur } & Kavdimatti & 16.494355 & 76.75099833 \\
\hline & & Shellagi & 16.54950167 & 76.65966333 \\
\hline \multirow[t]{4}{*}{ Chitradurga } & \multirow[t]{2}{*}{ Challakere } & Gowripura & 14.247728457 & 76.945109446 \\
\hline & & Parusharampura & 14.220265984 & 76.958669963 \\
\hline & \multirow[t]{2}{*}{ Hiriyur } & Devarkotta & 14.017957642 & 76.725594283 \\
\hline & & Kandikere & 14.109558 & 76.672411 \\
\hline \multirow[t]{4}{*}{ Tumkur } & Pavgada & Kottagudda & 14.08912 & 77.10233 \\
\hline & & Rajavanthi & 14.03515 & 77.16714 \\
\hline & Sira & Nejanthi & 14.008608 & 76.880164 \\
\hline & & Vajarahalli & 13.983925 & 76.8685 \\
\hline
\end{tabular}


Table.3 Incidence of stem and pod rot in major groundnut growing districts of Karnataka during kharif 2013

\begin{tabular}{|c|c|c|c|c|c|c|c|c|}
\hline District & Taluk & Village & $\begin{array}{l}\text { No. of } \\
\text { Fields }\end{array}$ & Variety & $\begin{array}{l}\text { Soil } \\
\text { type }\end{array}$ & DAS & $\begin{array}{l}\text { Stem } \\
\operatorname{rot}(\%)\end{array}$ & $\begin{array}{c}\text { Pod } \\
\operatorname{rot}(\%)\end{array}$ \\
\hline \multirow[t]{6}{*}{ Raichur } & \multirow[t]{2}{*}{ Raichur } & Chandrabanda & 3 & Local & Red & 75 & 33.3 & 45.4 \\
\hline & & Yapaldinni & 3 & Local & Red & $75-80$ & 25.3 & 37.5 \\
\hline & \multirow[t]{2}{*}{ Lingasugur } & Karadkal & 3 & TMV-2 & Red & 70 & 37.3 & 33.3 \\
\hline & & Eachanahal & 3 & TMV-2 & Red & 70 & 18.7 & 20.0 \\
\hline & \multirow[t]{2}{*}{ Deodurga } & Galag & 3 & TMV-2 & Red & $80-90$ & 17.6 & 11.1 \\
\hline & & Kajjibandi & 3 & TMV-2 & Red & $90-100$ & 21.2 & 25.0 \\
\hline \multicolumn{7}{|c|}{ Mean } & 25.57 & 28.65 \\
\hline \multirow[t]{6}{*}{ Koppal } & \multirow[t]{2}{*}{ Koppal } & Muddaballi & 3 & Local & Red & $90-100$ & 14.0 & 9.1 \\
\hline & & Halagera & 3 & Local & Red & $85-90$ & 10.7 & 7.7 \\
\hline & \multirow[t]{2}{*}{ Kushtagi } & Kanakoppa & 3 & GPBD 4 & Red & $90-95$ & 11.3 & 6.7 \\
\hline & & Byalihal & 3 & GPBD 4 & Red & $75-80$ & 17.5 & 0.0 \\
\hline & \multirow[t]{2}{*}{ Yelburga } & Bandi & 3 & JL-24 & $\begin{array}{c}\text { Red } \\
\text { sandy }\end{array}$ & $70-80$ & 26.7 & 33.3 \\
\hline & & Rajoor & 3 & JL-24 & $\begin{array}{c}\text { Red } \\
\text { sandy }\end{array}$ & $85-90$ & 22.4 & 27.3 \\
\hline \multicolumn{3}{|c|}{ Mean } & & & & & 17.19 & 14.00 \\
\hline \multirow[t]{6}{*}{ Ballari } & \multirow[t]{2}{*}{ Ballari } & Kakkabevinahalli & 3 & Local & $\begin{array}{l}\text { Red } \\
\text { sandy }\end{array}$ & $90-100$ & 38.5 & 25.0 \\
\hline & & Bommanahal & 3 & Local & $\begin{array}{c}\text { Red } \\
\text { sandy }\end{array}$ & $70-75$ & 29.6 & 16.7 \\
\hline & \multirow[t]{2}{*}{ Sirguppa } & Dasapur & 3 & Local & $\begin{array}{l}\text { Red } \\
\text { sandy }\end{array}$ & $75-80$ & 28.0 & 33.3 \\
\hline & & Konchigeri & 3 & Local & $\begin{array}{l}\text { Red } \\
\text { sandy }\end{array}$ & $80-85$ & 32.0 & 42.9 \\
\hline & \multirow[t]{2}{*}{ Kudligi } & Kudligi & 3 & Local & $\begin{array}{c}\text { Red } \\
\text { sandy }\end{array}$ & $90-95$ & 41.3 & 55.6 \\
\hline & & Sivapura & 3 & Local & $\begin{array}{c}\text { Red } \\
\text { sandy }\end{array}$ & $85-90$ & 46.7 & 40.0 \\
\hline \multicolumn{7}{|c|}{ Mean } & 31.61 & 35.56 \\
\hline \multirow[t]{4}{*}{ Gadag } & \multirow[t]{2}{*}{ Gadag } & Mulgund & 3 & Local & Red & $80-90$ & 7.1 & 0.0 \\
\hline & & Chinchalli & 3 & Local & Red & $80-90$ & 15.7 & 5.6 \\
\hline & \multirow[t]{2}{*}{ Ron } & Gajendragad & 3 & Local & Red & $90-100$ & 27.8 & 16.7 \\
\hline & & Goudageri & 3 & Local & Red & $70-80$ & 18.5 & 11.1 \\
\hline \multicolumn{7}{|c|}{ Mean } & 17.25 & 8.33 \\
\hline
\end{tabular}


Contd....

\begin{tabular}{|c|c|c|c|c|c|c|c|c|}
\hline District & Taluk & Village & $\begin{array}{l}\text { No. of } \\
\text { Fields }\end{array}$ & Variety & $\begin{array}{l}\text { Soil } \\
\text { type }\end{array}$ & DAS & $\begin{array}{l}\text { Stem } \\
\text { rot }(\%)\end{array}$ & Pod rot $(\%)$ \\
\hline \multirow[t]{4}{*}{ Kalaburgi } & \multirow[t]{2}{*}{ Kalaburgi } & Taj Sulthanpur & 3 & Local & Red & $80-90$ & 21.2 & 28.6 \\
\hline & & Kamalapur & 3 & Local & Red & $90-100$ & 30.6 & 46.7 \\
\hline & \multirow[t]{2}{*}{ Aland } & Aland rural & 3 & Local & Red & $75-80$ & 21.2 & 16.7 \\
\hline & & Telkuni & 3 & Local & Red & $70-80$ & 16.9 & 7.7 \\
\hline \multicolumn{7}{|c|}{ Mean } & 22.45 & 24.89 \\
\hline \multirow[t]{6}{*}{ Yadgir } & \multirow[t]{2}{*}{ Yadgir } & Ramsamudra & 3 & Local & Red & $70-80$ & 24.7 & 22.2 \\
\hline & & Hattikuni & 3 & Local & Red & $80-90$ & 21.2 & 12.5 \\
\hline & \multirow[t]{2}{*}{ Shahpur } & Bheemarayangudi & 3 & Local & Red & $90-95$ & 30.6 & 18.2 \\
\hline & & Gundapur & 3 & Local & Red & $90-100$ & 29.4 & 37.5 \\
\hline & \multirow[t]{2}{*}{ Shorapur } & Naganoor & 3 & Local & Red & $75-80$ & 38.7 & 40.0 \\
\hline & & Khanapur & 3 & Local & Red & $80-85$ & 32.9 & 23.5 \\
\hline \multicolumn{7}{|c|}{ Mean } & 29.56 & 25.65 \\
\hline \multirow[t]{4}{*}{$\begin{array}{c}\text { Chitradur } \\
\text { ga }\end{array}$} & \multirow[t]{2}{*}{ Challikere } & Challakere rural & 3 & Local & $\begin{array}{l}\text { Red } \\
\text { sandy }\end{array}$ & $80-85$ & 57.3 & 58.3 \\
\hline & & Nandanhalli & 3 & Local & $\begin{array}{l}\text { Red } \\
\text { sandy }\end{array}$ & $90-100$ & 54.7 & 63.6 \\
\hline & \multirow[t]{2}{*}{ Hiriyur } & Yarballi & 3 & Local & $\begin{array}{l}\text { Red } \\
\text { sandy }\end{array}$ & $90-95$ & 73.3 & 100.0 \\
\hline & & Hartikote & 3 & Local & $\begin{array}{l}\text { Red } \\
\text { sandy }\end{array}$ & $80-95$ & 65.9 & 72.7 \\
\hline \multicolumn{7}{|c|}{ Mean } & 62.80 & 73.67 \\
\hline \multirow[t]{4}{*}{ Tumkur } & \multirow[t]{2}{*}{ Pavagada } & $\begin{array}{l}\text { Kenchammana- } \\
\text { halli }\end{array}$ & 3 & Local & $\begin{array}{l}\text { Red } \\
\text { sandy }\end{array}$ & $80-90$ & 43.5 & 37.5 \\
\hline & & Lingadahalli & 3 & Local & $\begin{array}{c}\text { Red } \\
\text { sandy }\end{array}$ & $80-90$ & 52.9 & 46.2 \\
\hline & \multirow[t]{2}{*}{ Sira } & Dwaranakunte & 3 & Local & Red & $75-80$ & 42.7 & 41.7 \\
\hline & & Karehalli & 3 & Local & Red & $80-90$ & 31.3 & 37.5 \\
\hline & & Mean & & & & & 42.59 & 40.7 \\
\hline
\end{tabular}


Table.4 Incidence of stem and pod rot in major groundnut growing districts of Karnataka during kharif 2014

\begin{tabular}{|c|c|c|c|c|c|c|c|c|}
\hline District & Taluk & Village & $\begin{array}{l}\text { No. of } \\
\text { Field }\end{array}$ & Variety & $\begin{array}{l}\text { Soil } \\
\text { type }\end{array}$ & DAS & $\begin{array}{c}\text { Stem } \\
\operatorname{rot}(\%)\end{array}$ & $\begin{array}{c}\text { Pod } \\
\operatorname{rot}(\%)\end{array}$ \\
\hline \multirow[t]{6}{*}{ Raichur } & \multirow[t]{2}{*}{ Raichur } & Idapnur & 3 & TMV-2 & Red & $77-80$ & 33.8 & 38.5 \\
\hline & & Jambaldinni & 3 & Local & Red & $75-80$ & 42.9 & 33.3 \\
\hline & \multirow[t]{2}{*}{ Lingasugur } & Neeralkera & 3 & Local & Red & $85-90$ & 37.3 & 44.4 \\
\hline & & Bannigol & 3 & Local & Red & $80-85$ & 30.6 & 33.3 \\
\hline & \multirow[t]{2}{*}{ Deodurga } & Karadigudda & 3 & TMV-2 & Red & $80-90$ & 32.5 & 22.2 \\
\hline & & Gabbur & 3 & TMV-2 & Red & $90-100$ & 30.6 & 43.8 \\
\hline \multicolumn{7}{|c|}{ Mean } & 34.6 & 35.9 \\
\hline \multirow[t]{6}{*}{ Koppal } & \multirow[t]{2}{*}{ Koppal } & Indargi & 3 & Local & Red & $70-80$ & 21.3 & 18.2 \\
\hline & & Ginigera & 3 & Local & Red & $80-85$ & 15.7 & 8.3 \\
\hline & \multirow[t]{2}{*}{ Kushtagi } & Gumgeri & 3 & Local & Red & $70-75$ & 14.1 & 0.0 \\
\hline & & Ganganhal & 3 & Local & Red & $75-80$ & 10.8 & 0.0 \\
\hline & \multirow[t]{2}{*}{ Yelburga } & Talakal & 3 & JL 24 & Red & $65-70$ & 12.9 & 0.0 \\
\hline & & Boodagumpa & 3 & Local & Red & $70-75$ & 28.6 & 25.0 \\
\hline \multicolumn{7}{|c|}{ Mean } & 17.23 & 8.58 \\
\hline \multirow[t]{6}{*}{ Ballari } & \multirow[t]{2}{*}{ Ballari } & Bevinahalli & 3 & Local & Red & $70-80$ & 23.3 & 15.4 \\
\hline & & Upanayakanahali & 3 & Local & Red & $70-80$ & 32.2 & 17.7 \\
\hline & \multirow[t]{2}{*}{ Siruguppa } & Shanavasapur & 3 & Local & Red & $75-80$ & 25.3 & 11.1 \\
\hline & & Siddaramapura & 3 & Local & Red & $70-80$ & 30.5 & 21.1 \\
\hline & \multirow[t]{2}{*}{ Kudligi } & Bande basapur & 3 & Local & $\begin{array}{c}\text { Red } \\
\text { sandy }\end{array}$ & $75-85$ & 38.7 & 44.4 \\
\hline & & Hireheggadahal & 3 & Local & $\begin{array}{l}\text { Red } \\
\text { sandy }\end{array}$ & $75-85$ & 42.1 & 35.3 \\
\hline \multicolumn{7}{|c|}{ Mean } & 32.01 & 24.15 \\
\hline \multirow[t]{4}{*}{ Gadag } & \multirow[t]{2}{*}{ Gadag } & Seethalahari & 3 & Local & Red & $75-85$ & 17.8 & 15.4 \\
\hline & & Yalavatti & 3 & Local & Red & $75-80$ & 24.4 & 21.4 \\
\hline & \multirow[t]{2}{*}{ Ron } & Purthageri & 3 & Local & Red & $85-90$ & 13.3 & 6.3 \\
\hline & & Rajur & 3 & Local & Red & $75-80$ & 18.9 & 10.0 \\
\hline \multicolumn{7}{|c|}{ Mean } & 18.60 & 13.26 \\
\hline
\end{tabular}




\section{Contd....}

\begin{tabular}{|c|c|c|c|c|c|c|c|c|}
\hline District & Taluk & Village & $\begin{array}{l}\text { No. of } \\
\text { Fields }\end{array}$ & Variety & $\begin{array}{l}\text { Soil } \\
\text { type }\end{array}$ & DAS & $\begin{array}{l}\text { Stem } \\
\operatorname{rot}(\%)\end{array}$ & $\begin{array}{l}\text { Pod } \\
\operatorname{rot}(\%)\end{array}$ \\
\hline \multirow[t]{4}{*}{ Kalaburgi } & \multirow[t]{2}{*}{ Kalaburgi } & Wadagera & 3 & Local & Red & $65-85$ & 27.8 & 14.3 \\
\hline & & Tawargera & 3 & Local & Red & $70-85$ & 25.6 & 19.1 \\
\hline & \multirow[t]{2}{*}{ Aland } & Honhalli & 3 & Local & Red & $65-70$ & 14.7 & 4.2 \\
\hline & & Mamdapur & 3 & Local & Red & $70-80$ & 12.6 & 10.5 \\
\hline \multicolumn{7}{|c|}{ Mean } & 20.17 & 12.01 \\
\hline \multirow[t]{6}{*}{ Yadgir } & \multirow[t]{2}{*}{ Yadgir } & Anapoor & 3 & Local & Red & $80-90$ & 15.4 & 7.1 \\
\hline & & Bandalli & 3 & Local & Red & $70-80$ & 21.1 & 28.6 \\
\hline & \multirow[t]{2}{*}{ Shahpur } & Bhimanahalli & 3 & Local & Red & $90-100$ & 29.4 & 37.5 \\
\hline & & Raajaapoor & 3 & Local & Red & $70-80$ & 31.4 & 16.7 \\
\hline & \multirow[t]{2}{*}{ Shorapur } & Kavdimatti & 3 & Local & Red & $70-80$ & 30.0 & 42.9 \\
\hline & & Shellagi & 3 & Local & Red & $70-80$ & 36.7 & 42.9 \\
\hline \multicolumn{7}{|c|}{ Mean } & 27.33 & 29.27 \\
\hline \multirow[t]{4}{*}{$\begin{array}{c}\text { Chitradurg } \\
\text { a }\end{array}$} & \multirow[t]{2}{*}{ Challikere } & Gowripura & 3 & Local & $\begin{array}{l}\text { Red } \\
\text { sandy }\end{array}$ & $90-100$ & 55.3 & 75.0 \\
\hline & & Parusharampura & 3 & Local & $\begin{array}{l}\text { Red } \\
\text { sandy }\end{array}$ & $90-100$ & 57.7 & 64.3 \\
\hline & \multirow[t]{2}{*}{ Hiriyur } & Devarkotta & 3 & Local & $\begin{array}{l}\text { Red } \\
\text { sandy }\end{array}$ & $90-100$ & 62.7 & 85.7 \\
\hline & & Kandikere & 3 & Local & $\begin{array}{l}\text { Red } \\
\text { sandy }\end{array}$ & $80-85$ & 55.7 & 61.5 \\
\hline \multicolumn{7}{|c|}{ Mean } & 57.83 & 71.63 \\
\hline \multirow[t]{4}{*}{ Tumkur } & \multirow[t]{2}{*}{ Pavagada } & Kottagudda & 3 & Local & $\begin{array}{l}\text { Red } \\
\text { sandy }\end{array}$ & $70-85$ & 42.9 & 46.7 \\
\hline & & Rajavanthi & 3 & Local & $\begin{array}{l}\text { Red } \\
\text { sandy }\end{array}$ & $70-85$ & 53.8 & 58.3 \\
\hline & \multirow[t]{2}{*}{ Sira } & Nejanthi & 3 & Local & $\begin{array}{l}\text { Red } \\
\text { sandy }\end{array}$ & $85-90$ & 41.1 & 31.3 \\
\hline & & Vajarahalli & 3 & Local & $\begin{array}{l}\text { Red } \\
\text { sandy }\end{array}$ & $80-85$ & 46.7 & 57.1 \\
\hline \multicolumn{7}{|c|}{ Mean } & 46.09 & 48.34 \\
\hline
\end{tabular}


Table.5 Incidence of stem and pod rot of groundnut in parts of Karnataka during kharif 20132014 at different taluks

\begin{tabular}{|c|c|c|c|c|c|c|c|}
\hline \multirow[t]{2}{*}{ District } & \multirow[t]{2}{*}{ Taluk } & \multicolumn{2}{|c|}{ Stem rot $(\%)$} & \multirow{2}{*}{$\begin{array}{c}\text { Mean stem } \\
\text { rot }(\%) \\
\mathbf{2 0 1 3 - 1 4}\end{array}$} & \multicolumn{2}{|c|}{ Pod rot (\%) } & \multirow{2}{*}{$\begin{array}{c}\text { Mean pod rot } \\
(\%) \\
\mathbf{2 0 1 3 - 1 4}\end{array}$} \\
\hline & & 2013 & 2014 & & 2013 & 2014 & \\
\hline \multirow[t]{3}{*}{ Raichur } & Raichur & 29.33 & 38.30 & 33.81 & 41.45 & 35.89 & 38.67 \\
\hline & Lingasugur & 27.99 & 33.96 & 30.97 & 26.66 & 38.88 & 32.77 \\
\hline & Deodurga & 19.41 & 31.54 & 25.47 & 18.05 & 32.97 & 25.51 \\
\hline Mean & & 25.57 & 34.6 & 30.08 & 28.65 & 35.91 & 32.28 \\
\hline \multirow[t]{3}{*}{ Koppal } & Koppal & 12.67 & 18.52 & 15.59 & 8.39 & 13.25 & 10.82 \\
\hline & Kushtagi & 14.41 & 12.44 & 13.42 & 3.33 & 0.00 & 1.66 \\
\hline & Yelburga & 24.51 & 20.75 & 22.63 & 30.3 & 12.5 & 21.41 \\
\hline Mean & & 17.19 & 17.23 & 17.21 & 14.00 & 8.58 & 11.29 \\
\hline \multirow[t]{3}{*}{ Ballari } & Ballari & 34.04 & 27.77 & 30.90 & 20.83 & 16.51 & 18.67 \\
\hline & Sirguppa & 30.00 & 27.89 & 28.94 & 38.08 & 16.08 & 27.08 \\
\hline & Kudligi & 44.00 & 40.39 & 42.19 & 47.78 & 39.86 & 43.82 \\
\hline Mean & & 31.61 & 32.01 & 31.81 & 35.56 & 24.15 & 29.85 \\
\hline \multirow[t]{2}{*}{ Gadag } & Gadag & 11.38 & 21.11 & 16.24 & 2.78 & 18.4 & 10.59 \\
\hline & Ron & 23.13 & 16.11 & 19.62 & 13.89 & 8.12 & 11.00 \\
\hline Mean & & 17.25 & 18.60 & 17.93 & 8.33 & 13.26 & 10.79 \\
\hline \multirow[t]{2}{*}{ Kalaburgi } & Kalaburgi & 25.87 & 26.67 & 26.27 & 37.61 & 16.67 & 27.14 \\
\hline & Aland & 19.04 & 13.68 & 16.36 & 12.17 & 7.35 & 9.76 \\
\hline Mean & & 22.45 & 20.17 & 21.31 & 24.89 & 12.01 & 18.45 \\
\hline \multirow[t]{3}{*}{ Yadgir } & Yadgir & 22.94 & 18.24 & 20.59 & 17.36 & 17.85 & 17.60 \\
\hline & Shahapur & 30.00 & 30.42 & 30.21 & 27.85 & 27.08 & 27.46 \\
\hline & Shorapur & 35.76 & 33.33 & 34.54 & 31.76 & 42.88 & 37.32 \\
\hline Mean & & 29.56 & 27.33 & 28.44 & 25.65 & 29.27 & 27.46 \\
\hline \multirow[t]{3}{*}{ Chitradurga } & Challakere & 56.00 & 56.47 & 56.23 & 60.98 & 69.64 & 65.31 \\
\hline & Hiriyur & 69.61 & 59.19 & 64.44 & 86.36 & 73.62 & 79.99 \\
\hline & & 62.80 & 57.83 & 60.31 & 73.67 & 71.63 & 72.65 \\
\hline \multirow[t]{2}{*}{ Tumkur } & Pavgada & 48.23 & 48.30 & 48.26 & 41.82 & 52.51 & 47.16 \\
\hline & Sira & 36.96 & 43.89 & 40.42 & 39.58 & 44.19 & 41.88 \\
\hline Mean & & 42.59 & 46.09 & 44.34 & 40.70 & 48.34 & 44.52 \\
\hline \multicolumn{2}{|c|}{ Overall mean incidence } & 30.87 & 31.17 & 31.02 & 30.66 & 29.55 & 30.10 \\
\hline
\end{tabular}


Plate.1 Manifestation of symptoms of stem and pod rot of groundnut under field conditions

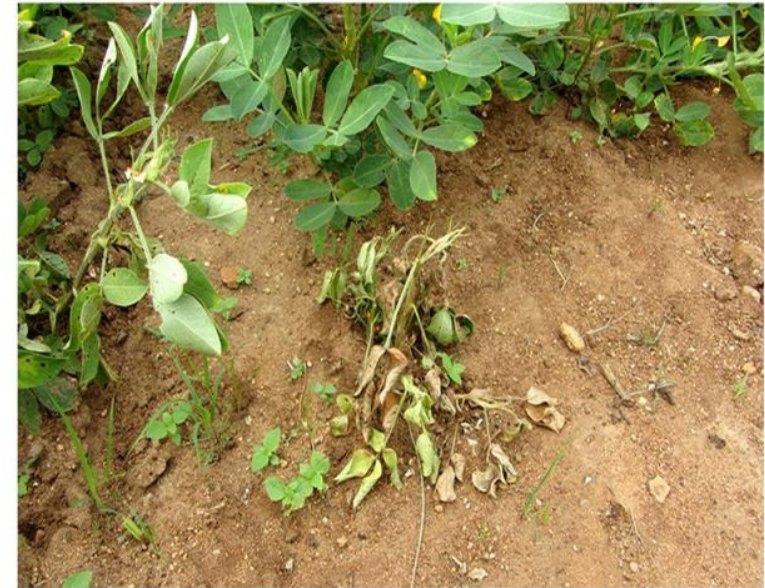

Yellowing and drooping of infected plant

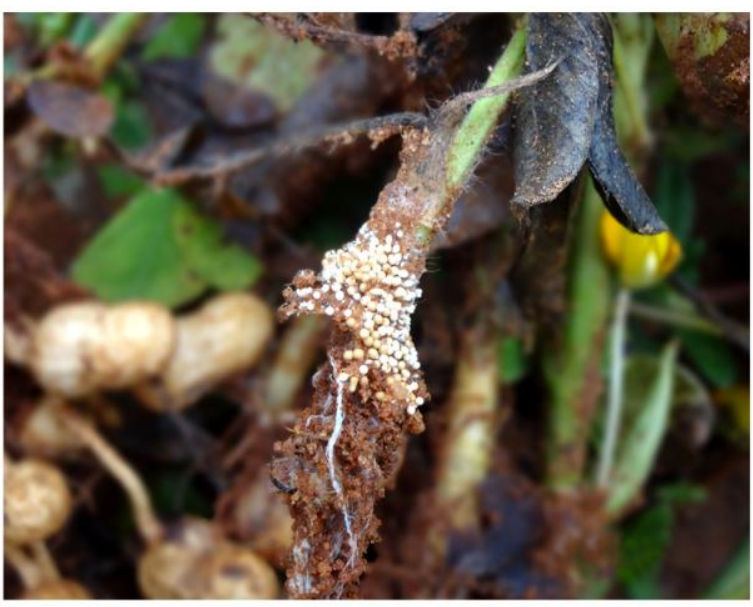

Sclerotial bodies on stem portion

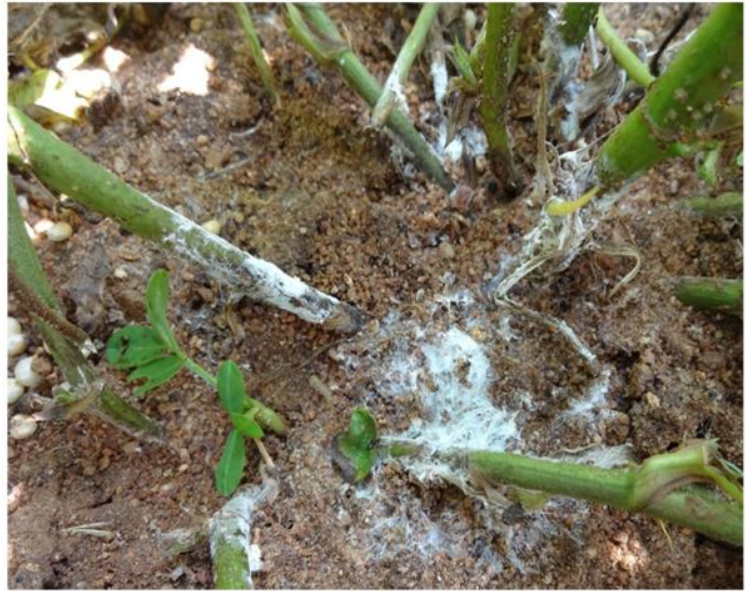

Mycelial mat on stem portion

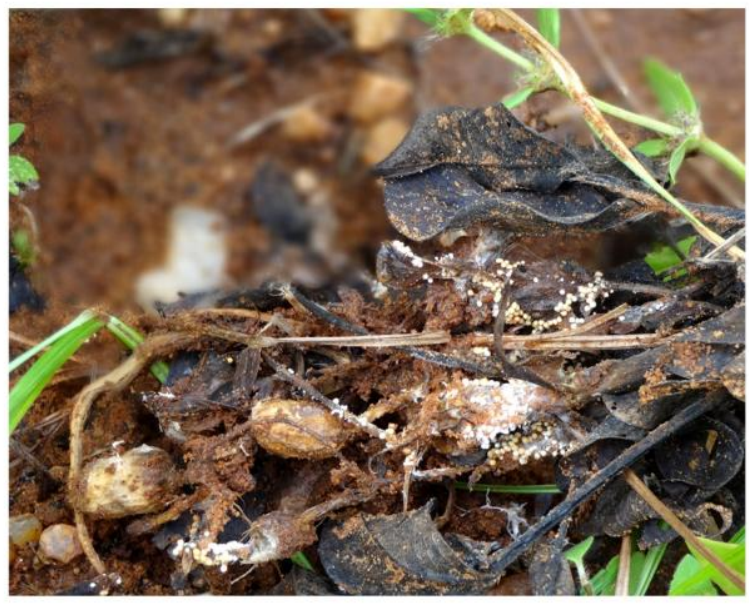

Mycelium and sclerotial bodies on pods

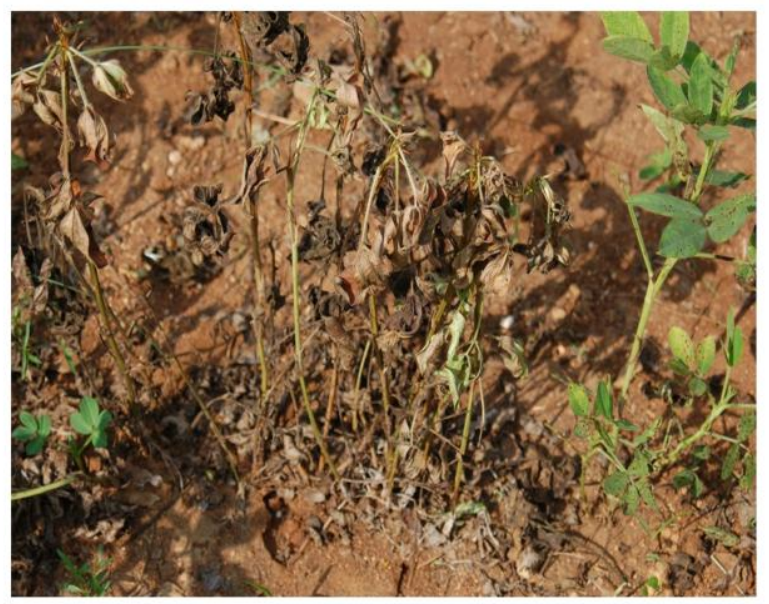

Wilting of plants 
Fig.1 GIS map showing incidence of stem and pod rot of groundnut during kharif 2013-14 in Raichur district
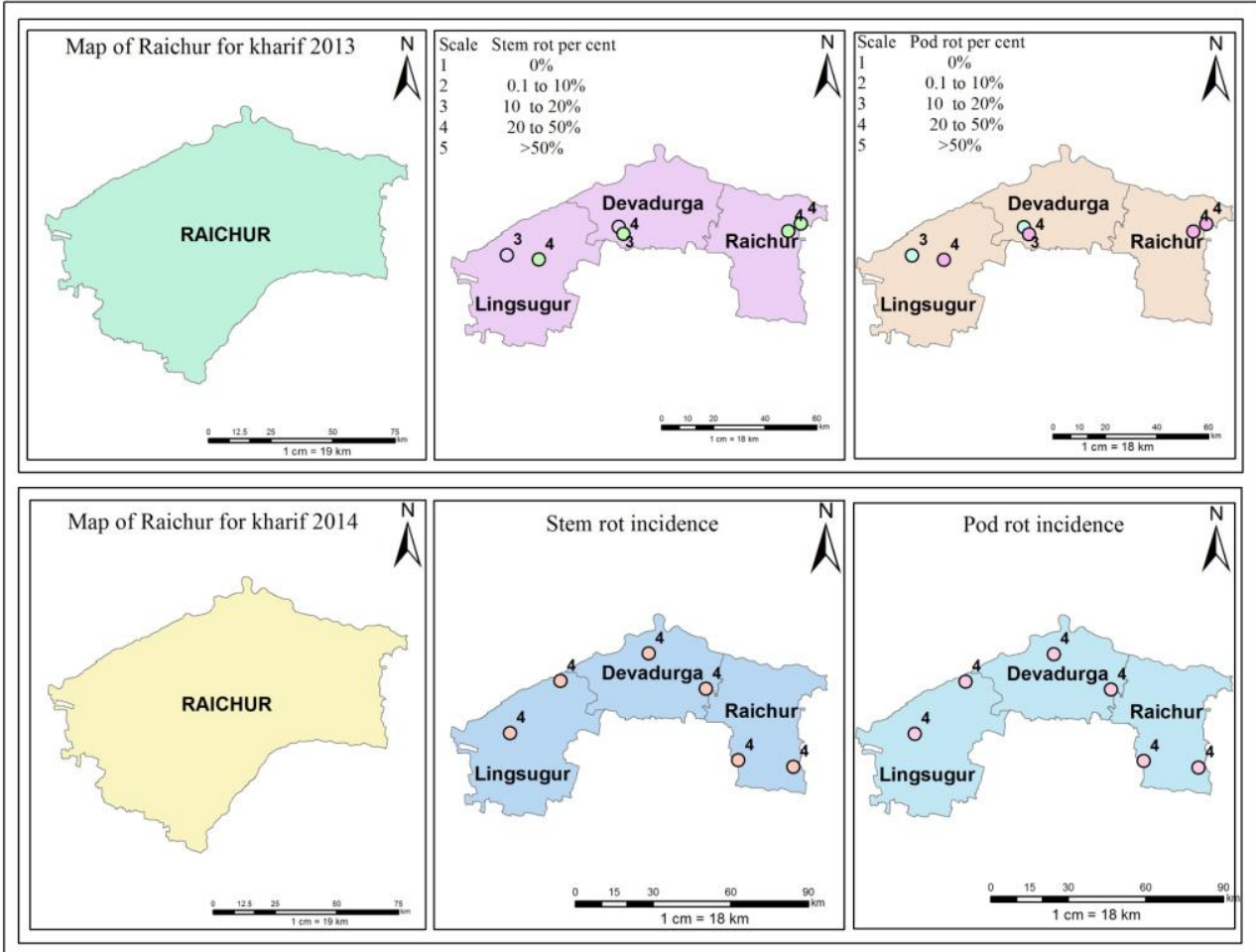

Fig.2 GIS map showing incidence of stem and pod rot of groundnut during kharif 2013-14 in Koppal district

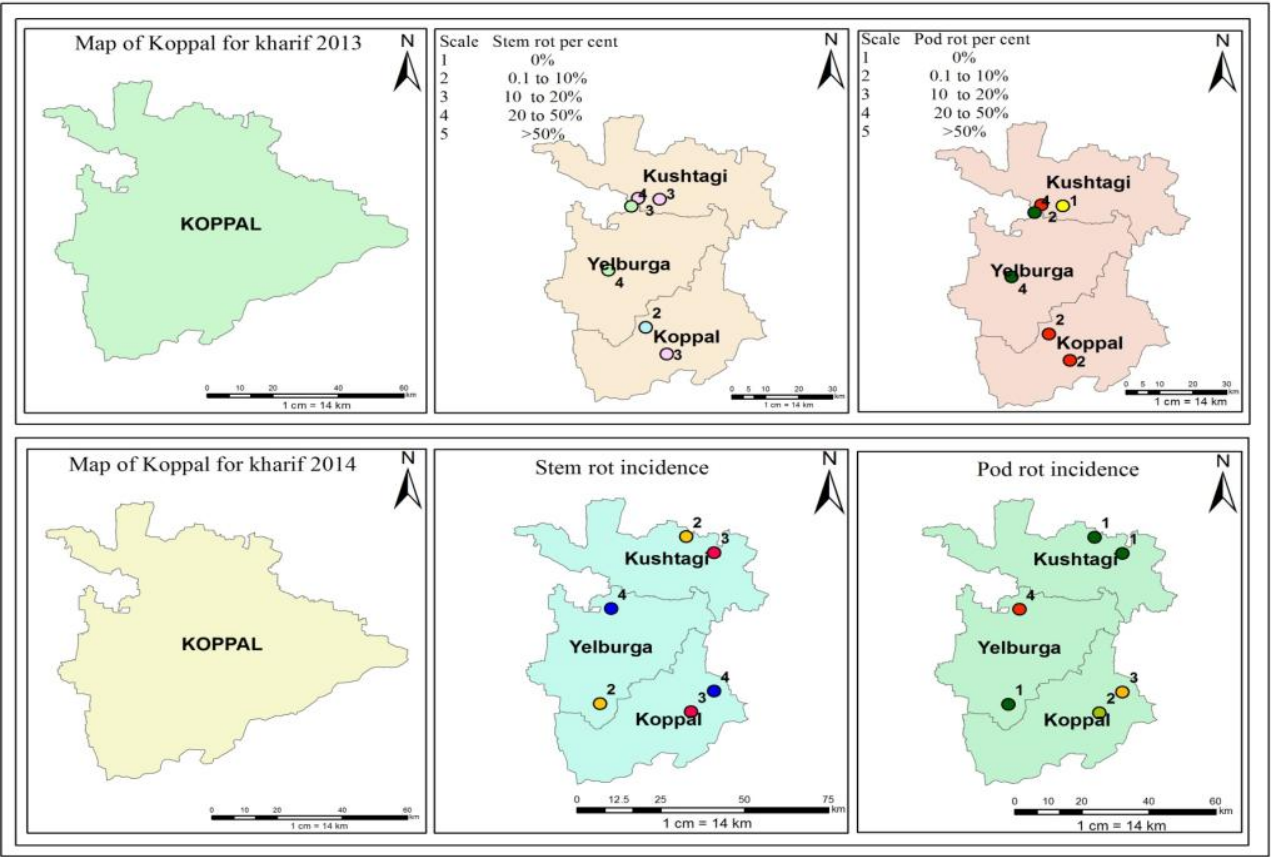


Fig.3 GIS map showing incidence of stem and pod rot of groundnut during kharif 2013-14 in Ballari district
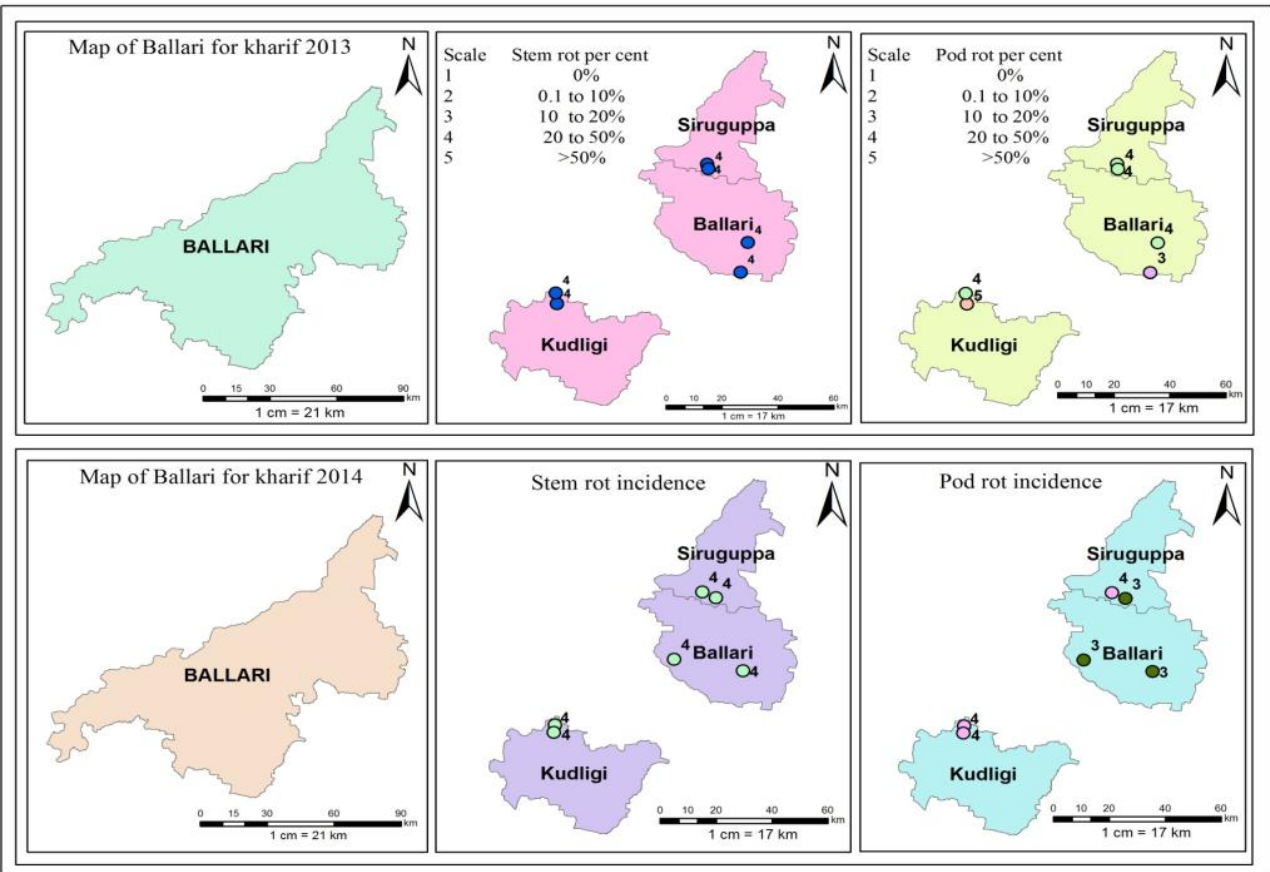

Fig.4 GIS map showing incidence of stem and pod rot of groundnut during kharif 2013-14 in Gadag district
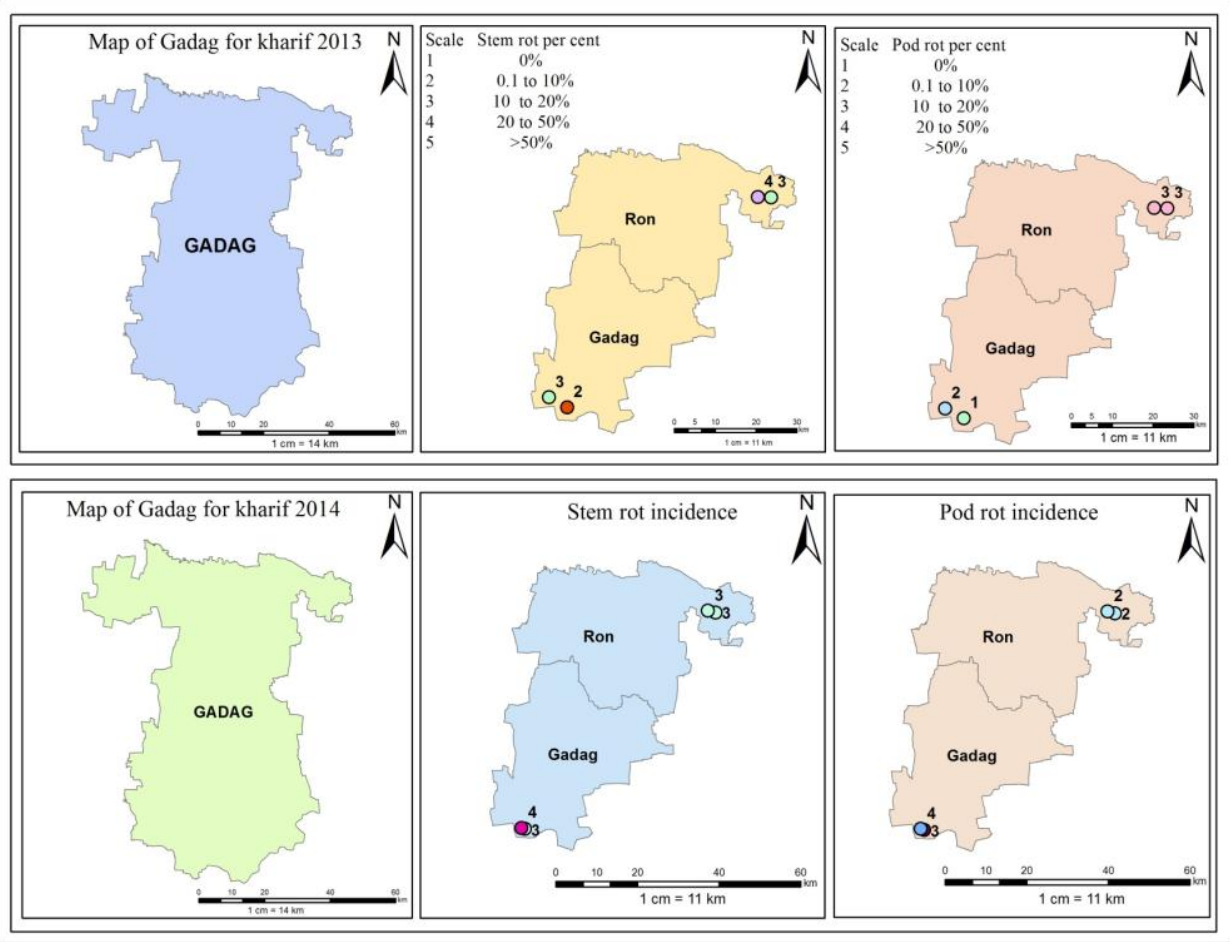
Fig.5 GIS map showing incidence of stem and pod rot of groundnut during kharif 2013-14 in Kalburgi district

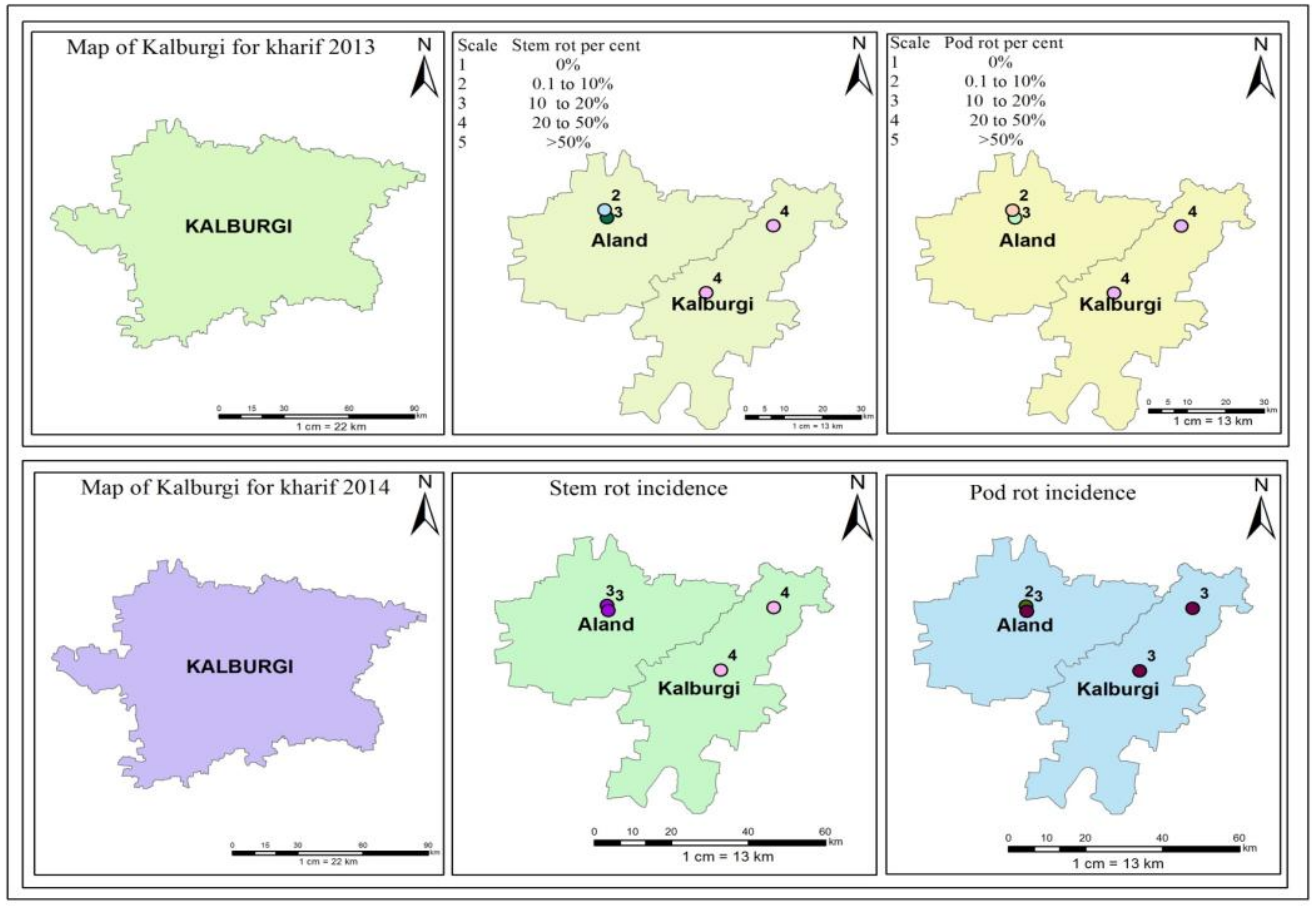

Fig.6 GIS map showing incidence of stem and pod rot of groundnut during kharif 2013-14 in Yadgir district

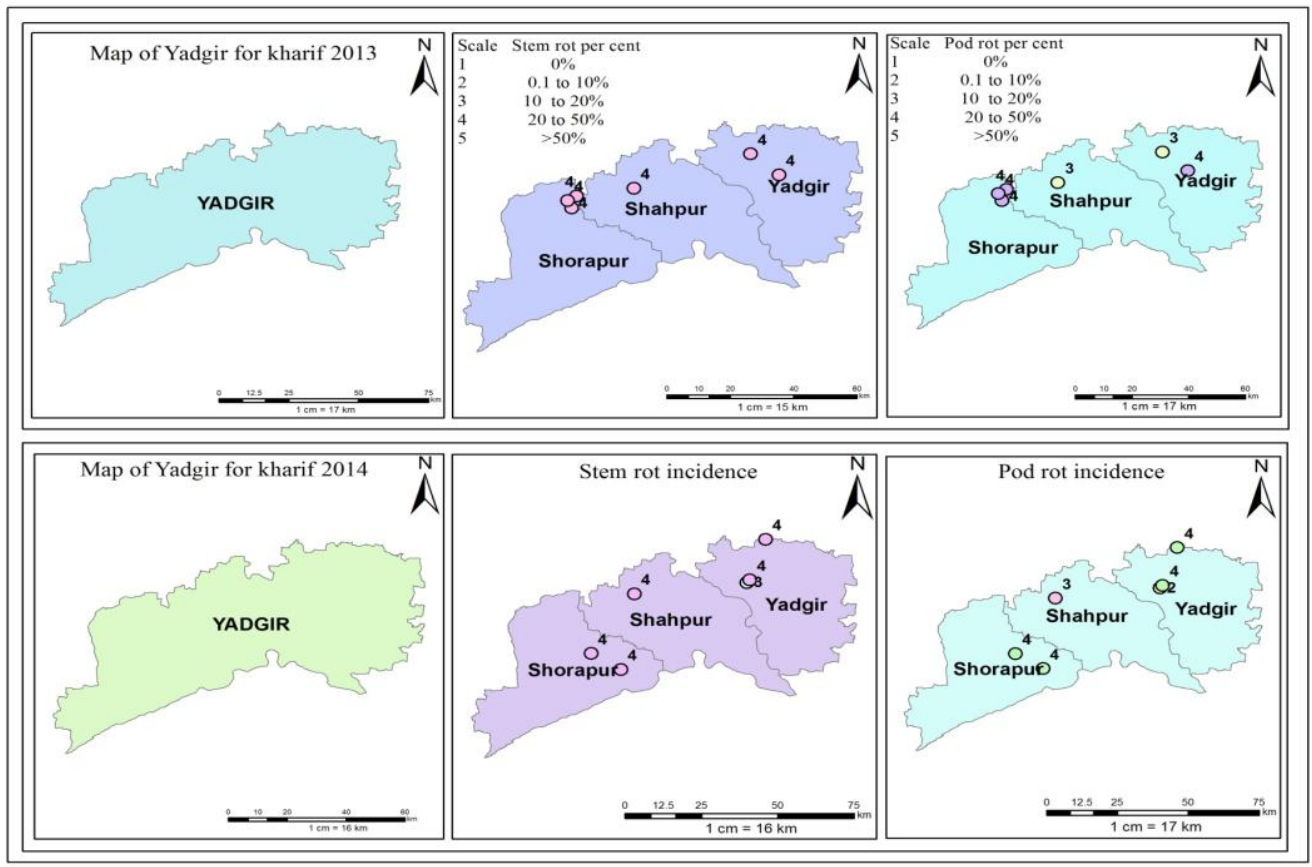


Fig.7 GIS map showing incidence of stem and pod rot of groundnut during kharif 2013-14 in Chitradurga district
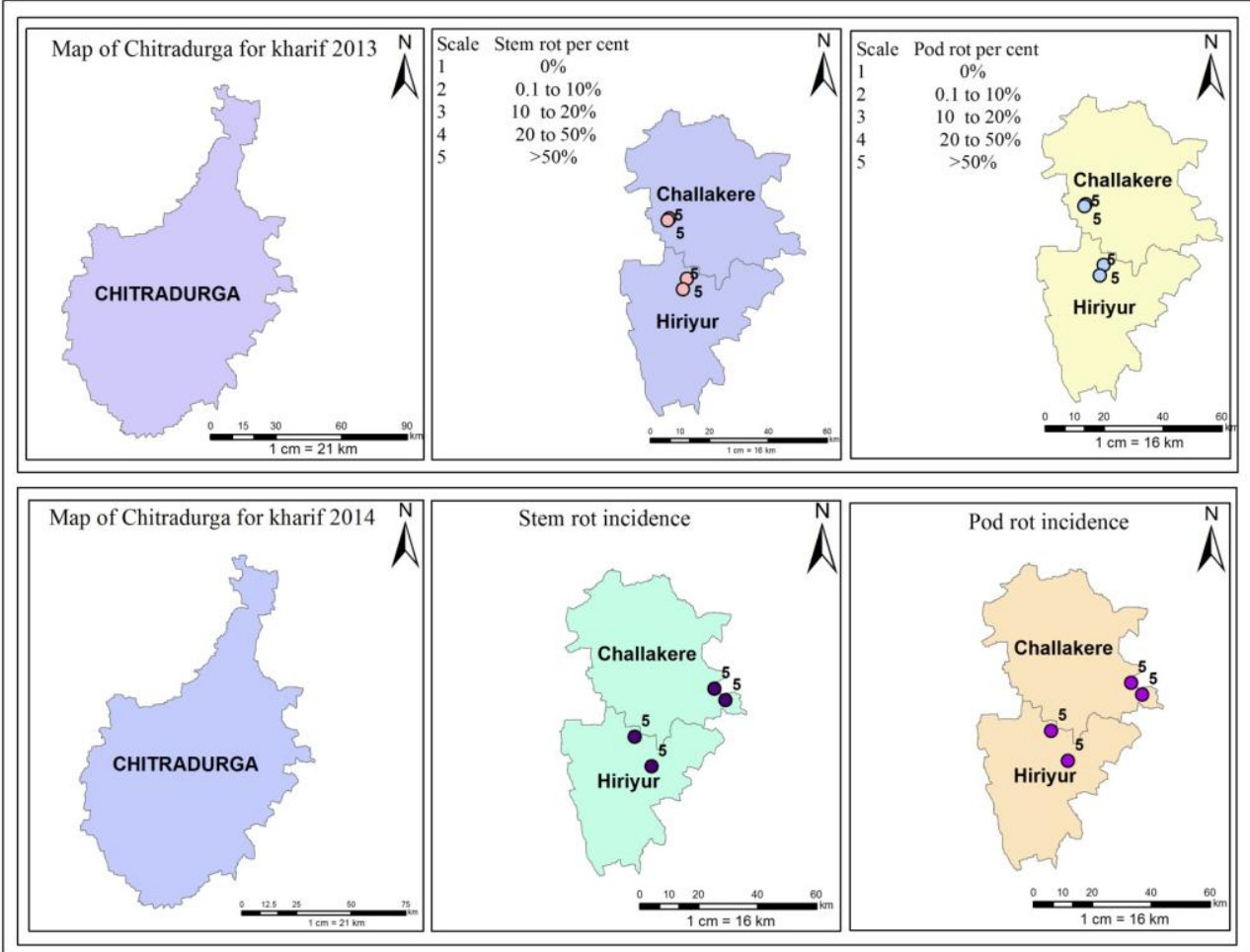

Fig.8 GIS map showing incidence of stem and pod rot of groundnut during kharif 2013-14 in Tumkur district
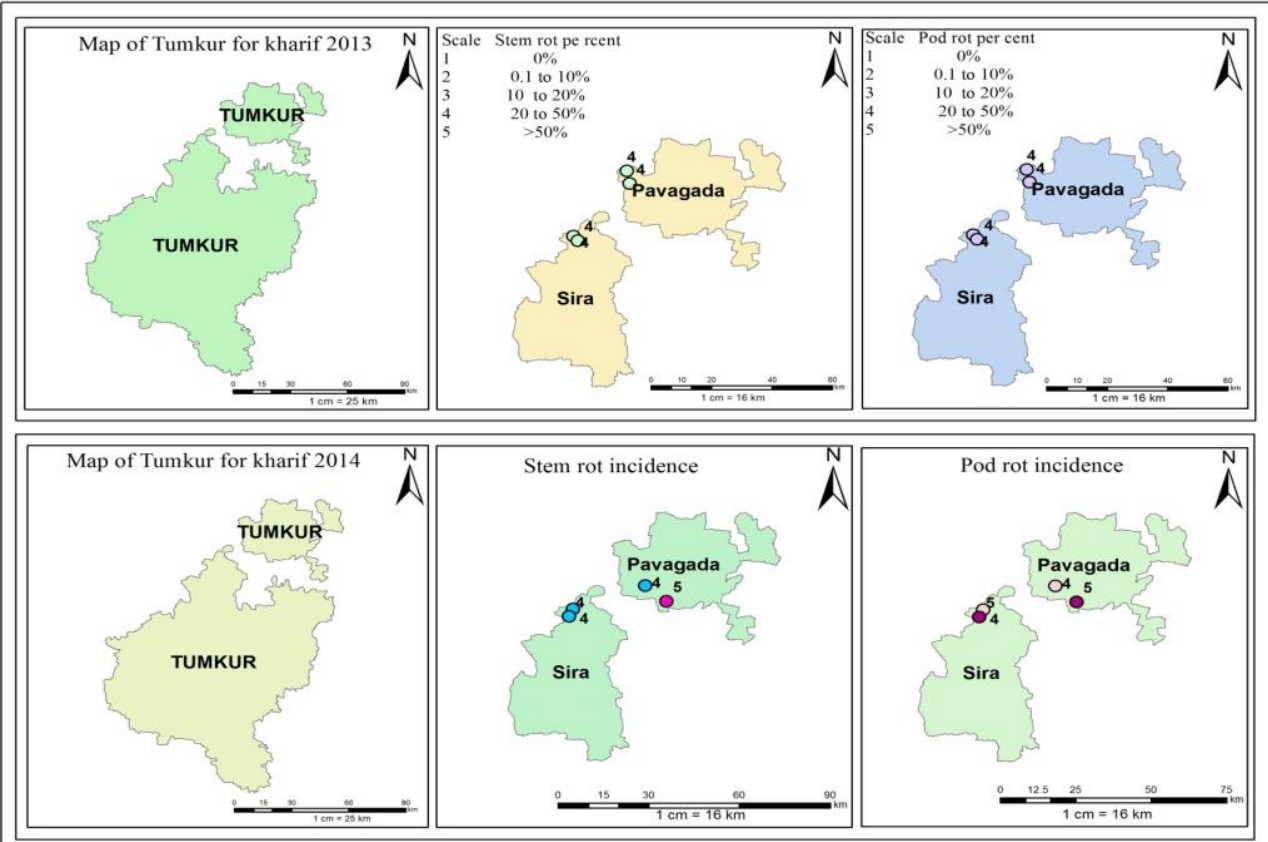
Continuous cultivation of any crop over the seasons and years will build up inoculum level to such an extent that the epidemic will become a common phenomenon (Chaube and Singh, 2001). It was noticed during survey that, the farmers of these districts are not practicing any management strategies or rotation of non host crops even though there was high incidence of stem and pod rot disease every year. Whereas the least incidence of stem and pod rot was recorded in Koppal district during the survey wherein we could able to observe cropping pattern such as maize-groundnut, sorghum-groundnut, bajragroundnut. Maize, sorghum and bajra crops were considered as non host crops for $S$. rolfsii it can be a one of the reason for less incidence of stem and pod rot disease in Koppal district. Anahosur (2001) also reported that maize, sorghum and bajra were free from the infection of $S$. rolfsii because the root exudates of these crops inhibit the germination of sclerotial bodies.

\section{References}

Anahosur KH. 2001. Integrated management of potato sclerotium wilt caused by Sclerotium rolfsii. Indian Phytopath 54: 158-166.

Anonymous 2014. Status paper on oilseeds, National Mission on Oilseeds and Oil Palm (NMOOP), New Delhi. pp. 24-25.

Chaube HS and Singh S. 2001. Introductory Plant Pathology Published by International Book Distributing Company, Lucknow, India. p. 195.

Karunakaran KR, Gracy CP, Lokesha H, Bantilan MCS, Charyalu DK, Rao PP, Rao, GDN, Valthiyalingan $\mathrm{M}$.
Venkataramana P. Upodhyoya HD, Janila P and Rao KPC. 2013. Groundnut baseline and early adoption surveys in South Asia, Synthesis report, ICRISAT Hyderabad. pp. 12-13.

Kulkarni V. 2007. Epidemiology and integrated management of potato wilt caused by Sclerotium rolfsii Sacc. Ph.D (Agri.) Thesis, Univ. Agric. Sci., Dharwad, Karnataka. pp. 89-91.

Nordern AJ, Smith OD, Gorbet DW. 1982. Breeding of cultivated peanut. (In Pattere HE and Youngs CT Eds.), Peanut Science and Technology. American Peanut Research and Education Society, Inc., Yoakun, Tex. pp. 95-122.

Pande S. and Rao JN. 2000. Changing scenario of groundnut diseases in Andhra Pradesh, Karnataka and Tamil Nadu states of India. Int Arachis Newsltr 20: 42.

Pande S, Rao J N, Reddy M V and Mcdonald D. 1994, Development of a greenhouse screening technique for stem rot resistance in groundnut. Int Arachis Newsltr 14: 2324.

Ramakrishna N. and Kolte S J 1988. Studies on pre-harvest groundnut pod rots. Indian Phytopath 41: 199-203.

Sennoi R, Jogloy S, Saksirirat W, Kesmala T, Singkham N. and Patanothai A. 2012. Levels of Sclerotium rolfsii inoculum influence identification of resistant genotypes in Jerusalem artichoke, African. J Microbiol Res 6(38): 6755-6760.

Siddaramaiah A L Krishnaprasad K S and Shivaram B N. 1979. Laboratory evaluation of fungicides against Sclerotium rolfsii Sacc. causing foot rot of groundnut. Pesticides, 13: 31-32.

\section{How to cite this article:}

Poornima, Gururaj Sunkad and Sudini, H. 2018. Status and Location of Hot Spots of Stem and Pod Rot of Groundnut in Karnataka, India. Int.J.Curr.Microbiol.App.Sci. 7(07): 3897-3912. doi: https://doi.org/10.20546/ijcmas.2018.707.454 